\title{
A new color-coded map of the $C$. elegans nervous system
}

Yemini, E. et al. Cell https://doi.org/10.1016/j.cell.2020.12.012 (2020)

The complete wiring diagram - or connectome - of the Caenorhabditis elegans nervous system was completed in 1986 by Sydney Brenner and colleagues who used electron microscope reconstruction to characterize the 302 neurons of the adult worm and hand-map all their connections. Since then, the C. elegans connectome has been widely used with microscopic images to identify neurons based on their morphology and position. However, the method remains uncertain and laborious, and more comprehensive and systematic approaches are needed for nervous system-wide neuronal identification. A study published in Cell describes a new multicolor fluorescence map that resolves all neuronal identities in the C. elegans nervous system by uniquely identifying each neuron by its color and position.

The team of investigators led by Eviatar Yemini from Oliver Hobert's lab at Columbia University created the map by designing a transgene called NeuroPAL (a neuronal polychromatic atlas of landmarks) that contains a combination of 40 neuron-specific promoters, each of which drives the expression of one or two of the three following fluorescent proteins: mTagBFP2, CyOFP1, and mNeptune2.5.

"The largest ganglia contain around 30 neurons. We reasoned that roughly 30 unique colors would be needed to reliably identify all neurons in each ganglion, and thus all neurons in the nervous system. Three spectrally distinct fluorophores, distinguishable at four or more different levels (high, medium, low, and undetectable), yield at least 64 different colors. Thus, three carefully chosen fluorophores should be enough to landmark the entire C. elegans nervous system," explain the investigators in their report.

The promoters used to build the transgene were selected from a candidate list of 133 published neuron-specific reporters. The investigators started with a small number of broadly expressed reporters and gradually expanded this initial set, adding and changing reporters to target

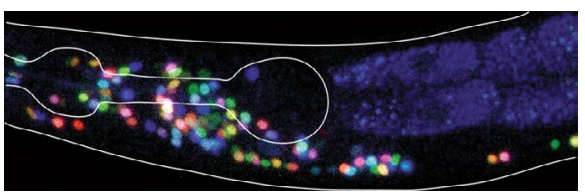

Neuronal cell identifications with NeuroPAL in C. elegans. Reprinted with permission from Reilly et al. (2020) Springer Nature.

progressively smaller subsets of neurons, until they found a final transgene composed of different reporter-fluorophore fusions that colored nearby neurons distinguishably in each ganglion.

The approach is fundamentally different from the previously described Brainbow method used to label individual neurons in different model organisms, including C. elegans. Whereas Brainbow is a stochastic multicolor labeling method, NeuroPAL assigns neurons a strictly deterministic color code based on the gene expression profile of the neuron, which is preserved across individuals. The NeuroPAL combination of reporters and colors also generates a more comprehensive color-coded atlas for $C$. elegans neurons than the system recently developed by Toyoshima and colleagues, which also combines different reporter-fluorophore fusions but only identifies $60 \%$ of all neurons in the C. elegans nervous system.

NeuroPAL can have multiple applications. Neurons labeled with NeuroPAL do not exhibit fluorescence in the green, cyan, or yellow emission channels, allowing the transgene to be used in combination with fluorescent reporters of gene expression or neural activity. In a study published in Nature in 2020, the Hobert Lab used the NeuroPAL landmark strain to analyze the expression pattern of all homeodomain proteins at single-neuron resolution in the C. elegans nervous system. Here, Yemini et al. used NeuroPAL to map the expression of metabotropic receptors for the neurotransmitters acetylcholine, GABA and glutamate. The investigators also used NeuroPAL to identify mediators of neuronal differentiation by crossing NeuroPAL worms with strains carrying null mutations of transcription factors and observing changes induced by the mutations in the color map. Finally, the team combined NeuroPAL with the $\mathrm{Ca}^{2+}$ indicator GCaMP6s to measure brain-wide responses to attractive and repulsive chemosensory cues. They showed that each stimulus activated a different set of neurons and interneurons, many of which had no previously known function. These findings suggest that NeuroPAL could be a useful tool for future experiments aiming to elucidate the relationship between circuit structure and function.

In addition, Yemini and colleagues developed a software pipeline to partially automate the annotation of neuronal identities in NeuroPAL images (https://www. hobertlab.org/neuropal/). The pipeline, which estimates a neuron's identity using a statistical atlas of NeuroPAL colors and positions, proved to be $86 \%$ and $94 \%$ accurate for neurons in the head and the tail, respectively. "Our algorithm also provided a means of assessing the importance of color information in assigning cell identities. When we restricted the model to assign identities only on the basis of location, automated accuracy dropped to $50 \%$ for the head and $68 \%$ for the tail. These results confirm a substantial improvement in accuracy with the color information provided by NeuroPAL," write the investigators.

The investigators also suggest that NeuroPAL could be extended to other genetically tractable animals such as fruit fly and zebrafish, and provide a software to guide the choice of reporterfluorophore combinations for other tissues and organisms.

Alexandra Le Bras

Published online: 18 January 2021

https://doi.org/10.1038/s41684-021-00710-5 